\title{
A Third Generation Computer Interface for Ultrasonic Pulse Echo Nondestructive Testing
}

\author{
G. C. Stoker
}

Prepared by Sandia Laboratories, Albuquerque. Nerw Mexico 87115

\section{Sandia Laboratories}

When printing a copy of any digitized SAND Report, you are required to update the markings to current standards. 
Issued by Sandia Laboratories, operated for the United States Energy

Research and Development Administration by Sandia Corporation.

\section{NOTICE}

This report was prepared as an account of work sponsored by the United States Government. Neither the United States nor the United States Energy Research and Development Administration, nor any of their employees, nor any of their contractors, subcontractors, or their employees, makes any warranty, express or implied, or assumes any legal liability or responsibility for the accuracy, completeness or usefulness of any information, apparatus, product or process disclosed, or represents that its use would not infringe privately owned rights. 
SAND75-0056

Unlimited Release

Published February 1975

\title{
A THIRD GENERATION COMPUTER INTERFACE FOR ULTRASONIC PULSE ECHO NONDESTRUCT IVE TESTING
}

G. C. Stoker

NDT Technology Division 9352

\begin{abstract}
A third generation computer interface is described in detail. Echo Trap III as it is called interfaces standard ultrasonic pulse echo/through transmission units with minicomputer systems. This approach allows computerized data acquisition and analysis for improved NDT results. Brief results are included.
\end{abstract}


A THIRD GENERATION COMPUTER

INTERFACE FOR ULTRASONIC PULSE ECHO

NONDESTRUCT IVE TEST ING

\section{Introduction}

Ultrasonic pulse echo testing techniques are widely used in the field of nondestructive testing. Maximum utilization of these techniques has only recently been realized using computer based, fully automated scanning systems. In order to efficiently use a system of this type, it is first necessary to electronically preprocess the basic ultrasonic signals to get the information they contain into a suitable form for computer data recording and analysis. The interface being described performs this function and represents a third generation development which includes improvements over previously developed first and second generation instruments.

\section{Background}

The basic ultrasonic pulse echo signal is generated in the following way. An ultrasonic transducer (usually a piezoelectric device) is first stimulated by an electronic signal or pulse. This causes the transducer to emit ultrasonic waves in the form of short bursts of ultrasonic energy at the resonant frequency of the transducer. These waves are then propagated through the material being tested. As the waves propagate through the material, any anomalies or flaws encountered will reflect a percentage of the energy in the wave. This reflected energy (or echo) propagates back through the material and is received, usually, by the same 
transducer that generated the initial wave. The sum of the time it takes for the wave to travel to an anomaly plus the time it takes the echo to return is a function of the depth of the anomaly in the material. The amplitude of the received echo is a function of the severity of the anomaly. These are the two most important parameters of the echo signal.

Figure 1 shows a typical pulse echo immersion setup with four associated waveforms. The waveform at $A$ is the actual transducer voltage and input signal for the receiver. This signal is amplified in the receiver and passed through an absolute value circuit or detector. The output of this circuit shown at $B$ is further amplified and then filtered slightly to smooth its envelope. Waveform $\mathrm{C}$ is the resulting final video output. This is also the assumed form for all video signals described in this report. Lastly, waveform $D$ is the IOI signal (interval of interest) and is derived from the unblanking signal of the CRT display in the receiver. The purpose of this signal will be described later.

The first generation interface instrument used a "gate," or series of gates synchronously timed to, and appropriately placed within, the interval of interest in order to interpret the video signal. If an echo is received during one of these gates, its amplitude is measured using conventional analog peak detection circuits. Furthermore, since the time delay to the gate receiving the echo is usually known, the approximate elapsed time to the echo itself is known. There are, however, problems associated with this technique. In order to attain reason- 

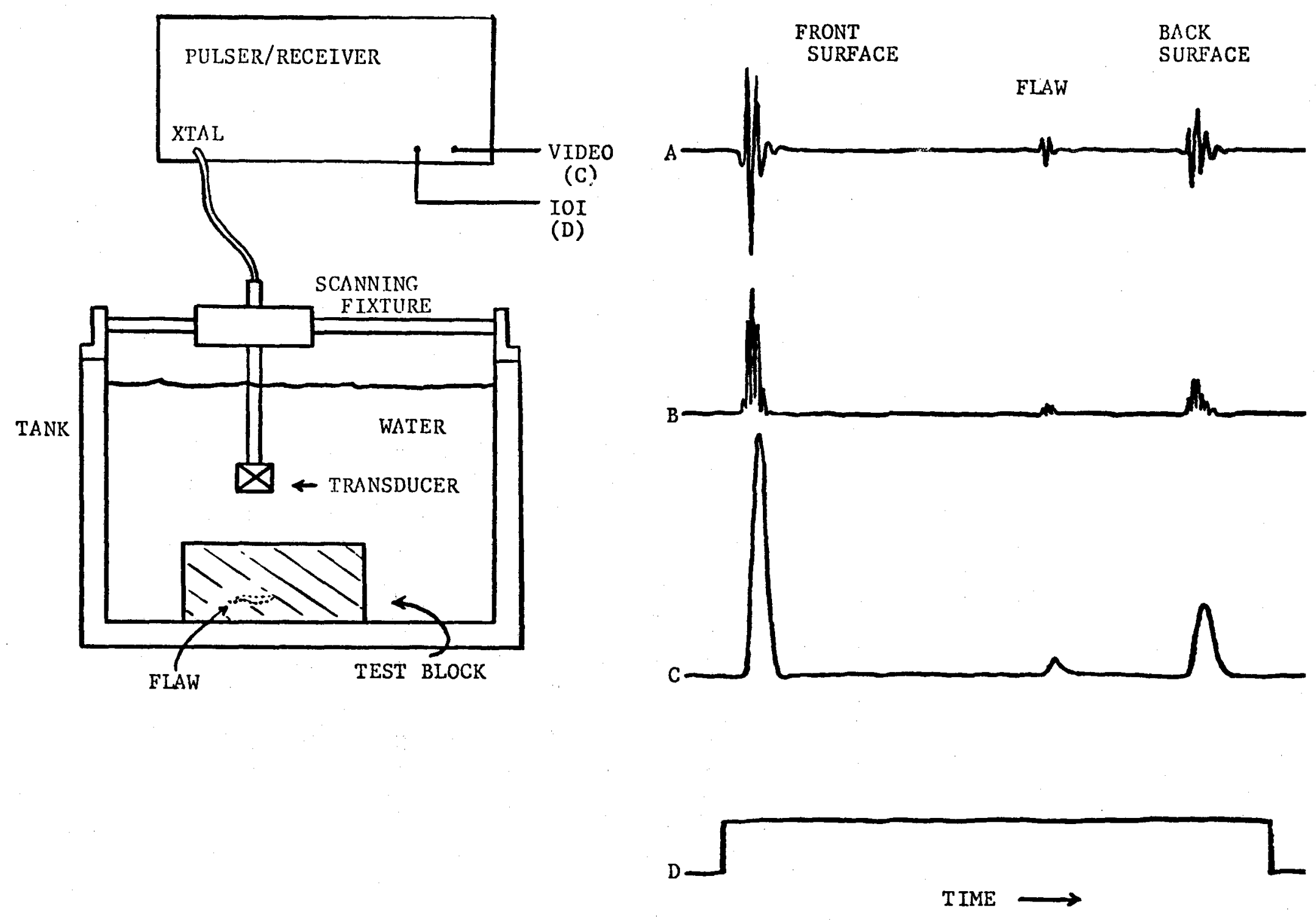

Figure 1. Pulse Echo System 
able time resolution, it is necessary to use many short duration gates. This not only complicates the circuitry, but increases the probability that a gate or gates will split an echo. If one echo is received for a time longer than one gate, it may erroneously be interpreted as more than one echo.

The second generation interface instrument, within limits, solved these problems. Using an amplitude threshold detector, the echo signal itself generates the gate for the peak sensing circuitry. This basically asynchronous approach insures that the echo signal is always centered in the gate and that the gate is as wide as the echo, allowing reasonable amplitude analysis regardless of when an echo is received. Time (or depth) information is obtained using a linear ramp that is started with either the stimulation pulse or the echo from the front surface of the part, depending on the type of test. The voltage attained by the ramp generator when the threshold is exceeded by an echo is proportional to the total delay time. Many peak detection circuits can be used to allow for as many echoes per stimulation pulse as desired. All peak values are held long enough for an A/D converter in the computer system to read them.

Still, a problem with these techniques is that accuracy in the peak detection circuitry is difficult to achieve, especially as the duration and amplitude of the echo peaks decrease. Using well damped transducers with resonant frequencies of $10 \mathrm{mHz}$ and higher, total echo widths of 50 to 200 ns are routinely received. These signals typically spend only 10 to 40 ns within $90 \%$ of 
their true peak. These are very taxing circumstances for conventional peak detectors to handle.

Also, the ultimate value of the time ramp has to be held with either a peak detector, or some kind of integrator technique. In either case, offsets, drift, and ramp nonlinearities all become sources of error.

A third generation ultrasonic interface has been built and appropriately named Echo Trap III. This device eliminates the problems encountered in second generation devices and is easily expandable for greater resolution or more channels.

\section{$\underline{\text { Requirements }}$}

Knowing that this third generation interface would be almost completely digital, some consideration of the requirements was in order. First of all, the number of flaws that may be detected with any one transducer stimulation pulse is normally quite low. Each flaw can be considered as a mask to any additional flaws that lie beneath it. Each successively deeper flaw requires a separate channel for analysis and recording. If the number of channels is a whole integer power of two, the output multiplexing process is quite simple using a binary counter to address the channels. Because of this, four flaw channels are used in Echo Trap III and have been found to be quite adequate.

It was initially postulated that high echo amplitude resolution would be required but experience with the second generation interfaces has shown otherwise. Small geometric variations from 
test to test make it very difficult to insure amplitude accuracy of better than a few percent. Also, because of the extremely short duration of some of the echoes (less than $50 \mathrm{~ns}$ ), a very high speed A/D converter would be necessary to digitize the signal. Since resolution and speed are basically opposing parameters of an A/D converter, some compromise was necessary. A special 4-bit binary A/D converter was built and is described in more detail later. Although this allows only 15 flaw amplitude levels, it has proven to be very satisfactory.

In order to make reasonably accurate sound velocity measurements and to locate flaws accurately, it was desired to have time resolution on the order of $0.1 \%$ full scale. Therefore, 10-bit binary counters were used in Echo Trap III. Although greater resolution is easily obtained, the accuracy of these readings could be questioned. This is because the echo time gates are determined by the echo signals themselves as they exceed the threshold level. Since the threshold is an operator variable, and the echo signal risetime is a function of the specific test, the actual elapsed time to each echo is difficult to define.

In order to maintain maximum bit resolution over a broad range of delay times, a time base selector is provided. This allows operation from $25 \mathrm{usec}$ full scale to $400 \mathrm{usec}$ full scale in appropriate steps. 


\section{General Description}

Figure 2 shows a general block diagram for the interface. The lower portion of the figure shows a typical video signal which can be used as a reference for this description. This video signal is seen entering a 4-bit peak latching $A / D$ converter. Threshold information is derived in this converter and is used by the control logic. The IOI signal also goes to the control logic,

At time $t_{0}$, the IOI signal rises to alert the control that video pulses are coming. At time $t_{1}$ the first echo (front surface) crosses the threshold and this starts all four counters at whatever timebase rate has been selected by the operator. At time $t_{2}$ the first flaw echo crosses the threshold and causes the control to stop Counter 1 which now holds the elapsed time to flaw number one. At times $t_{3}, t_{4}$, and $t_{5}$, Counters 2,3 , and 4 respectively are stopped and these counters now hold the elapsed times for receival of signals from flaws two, three, and four. The control block and peak latching converter were also determining the peak amplitudes of the flaws and storing them in their respective latches at the same time that the elapsed time data was being determined. At time $t_{6}$, the IOI ends and the multiplexing cycle begins. The multiplexer will, under computer control, place the amplitude and time of each flaw on the output data bus in a sequential manner. After this is done, the control block will reset the counters and wait for the next IOI pulse. 

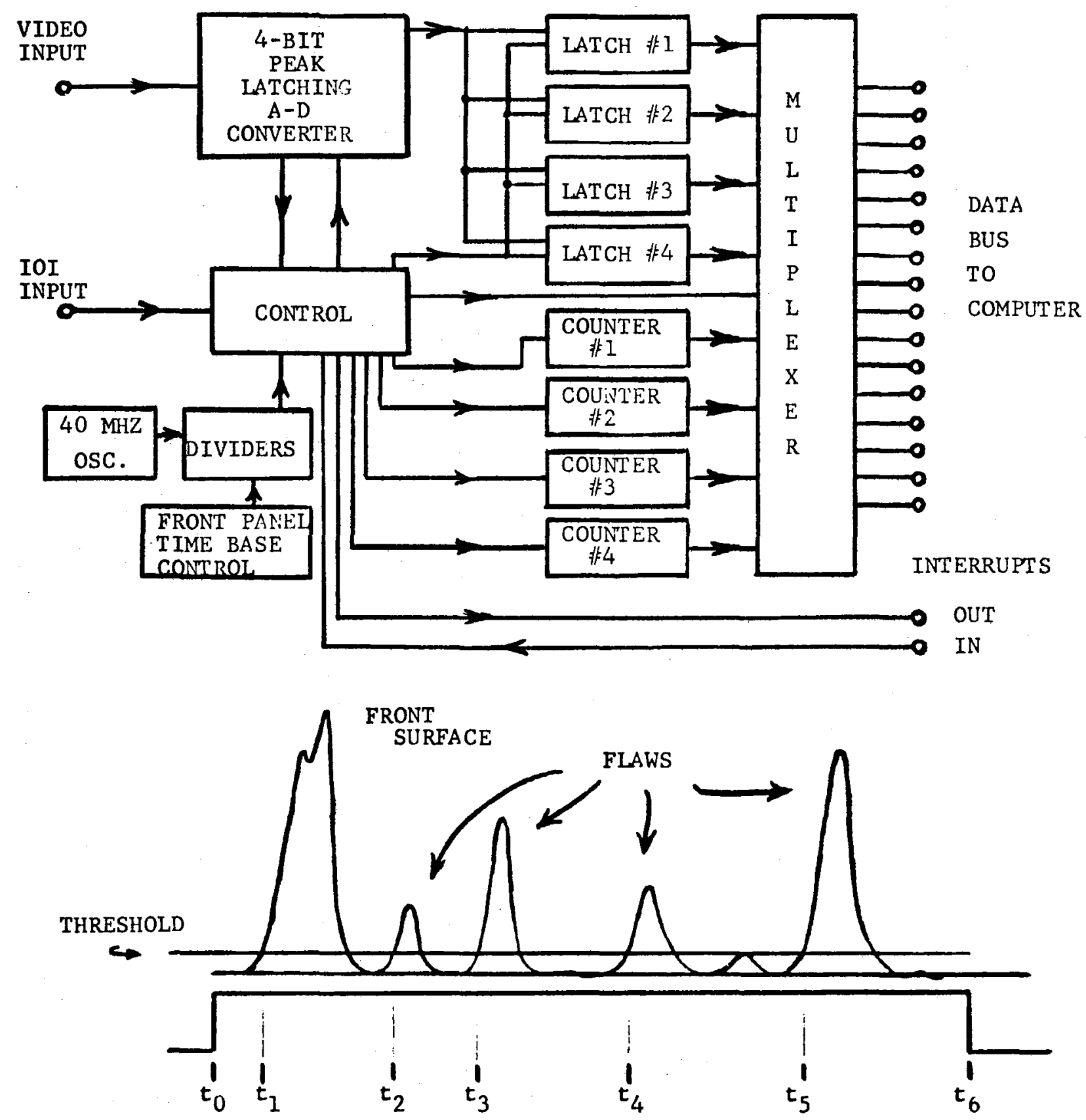

Figure 2. Interface Block Diagram 


\section{$\underline{\text { Results }}$}

This interface (Echo Trap III) has been used between a reflectoscope ultrasonic immersion system and a PDP-11/20 minicomputer system. The interface operation was checked by using standard blocks with flat bottomed holes. Figure 3 shows a plot of the location and depth of the holes in a test block. The interface shows complete repeatability in data acquisition other than setup variations which are a function of the operator.

The remainder of this report is devoted to a detailed description of the operation of Echo Trap III.

The Interface in Detail

Echo Trap III can be driven by almost any commercial ultrasonic receiver. Only two outputs from the receiver are needed. These may have to be added, however, depending on the instrument selected. These outputs are video and IOI (Interval of Interest). The video signal contains the ultrasonic echo information. The IOI signal defines the time interval being viewed on the receiver's display. Echo Trap III requires both of these signals to be zero referenced and positive going. Video amplitude shall be $+150 \mathrm{mV}$ for full scale. IOI amplitude shall be $+300 \mathrm{mV}$.

It is the operators responsibility to assure that the first echo seen on the display is the front surface of the part being tested. This echo will not be treated as a flaw, but will be used as a timing reference for all following echoes. This allows the time information output from the interface to be used to determine the depth of flaws below the front surface. 


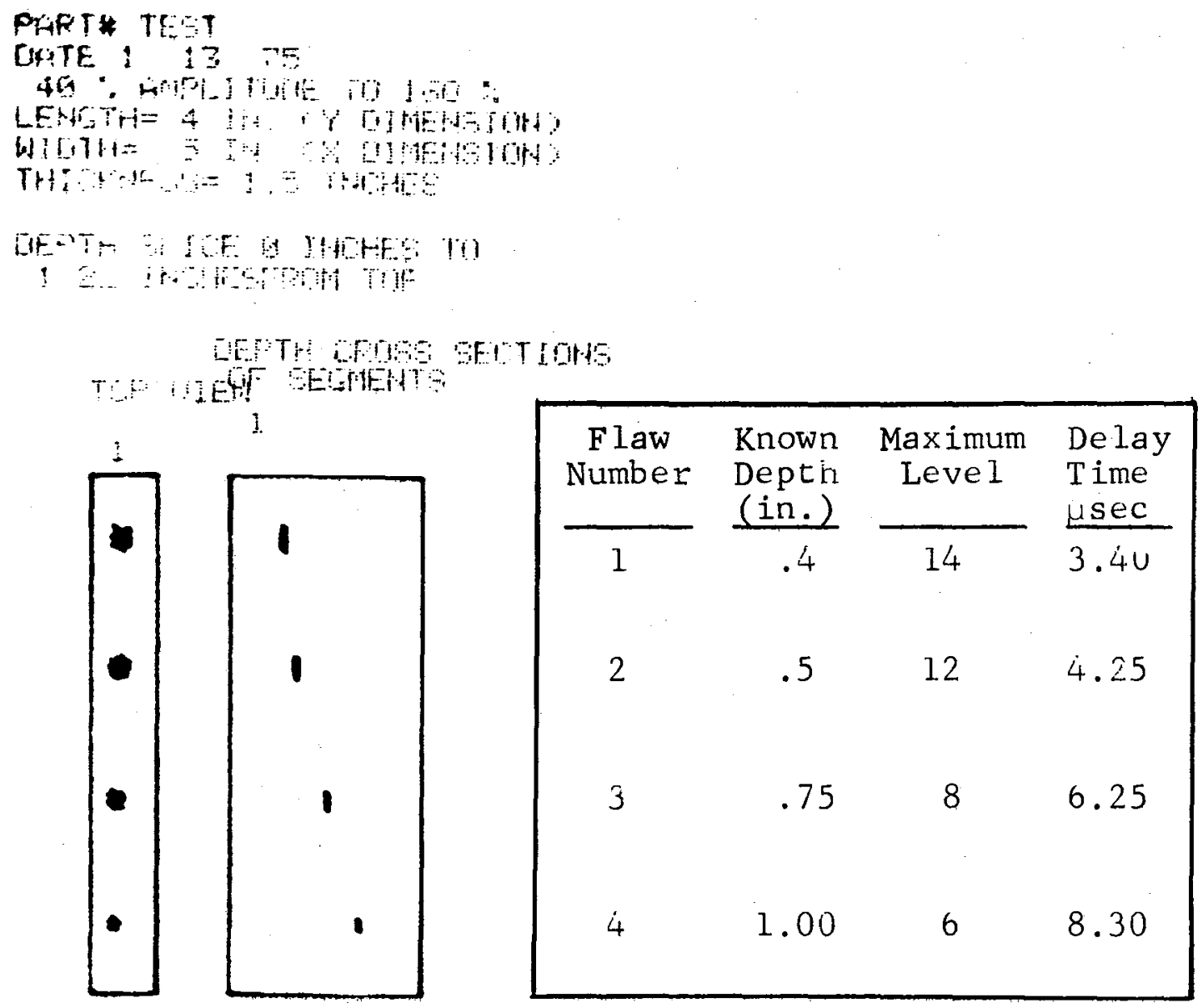

Figure 3. Data Plot

In the interface, the video signal goes through an ac coupled wideband amplifier and clamping circuit. This raises the full scale level to $1.5 \mathrm{~V}$ and assures a zero referenced base line. As shown in Fig. 4 the amplifier is a two-stage cascaded design using a 741 type op-amp as a clamping control. The IOI signal also goes through an amplifier shown in Fig. 5. This is a single stage, inverting amplifier, with its output clamped to the +5 VDC logic level. Except for power supplies, these circuits are the extent of analog signal processing. 


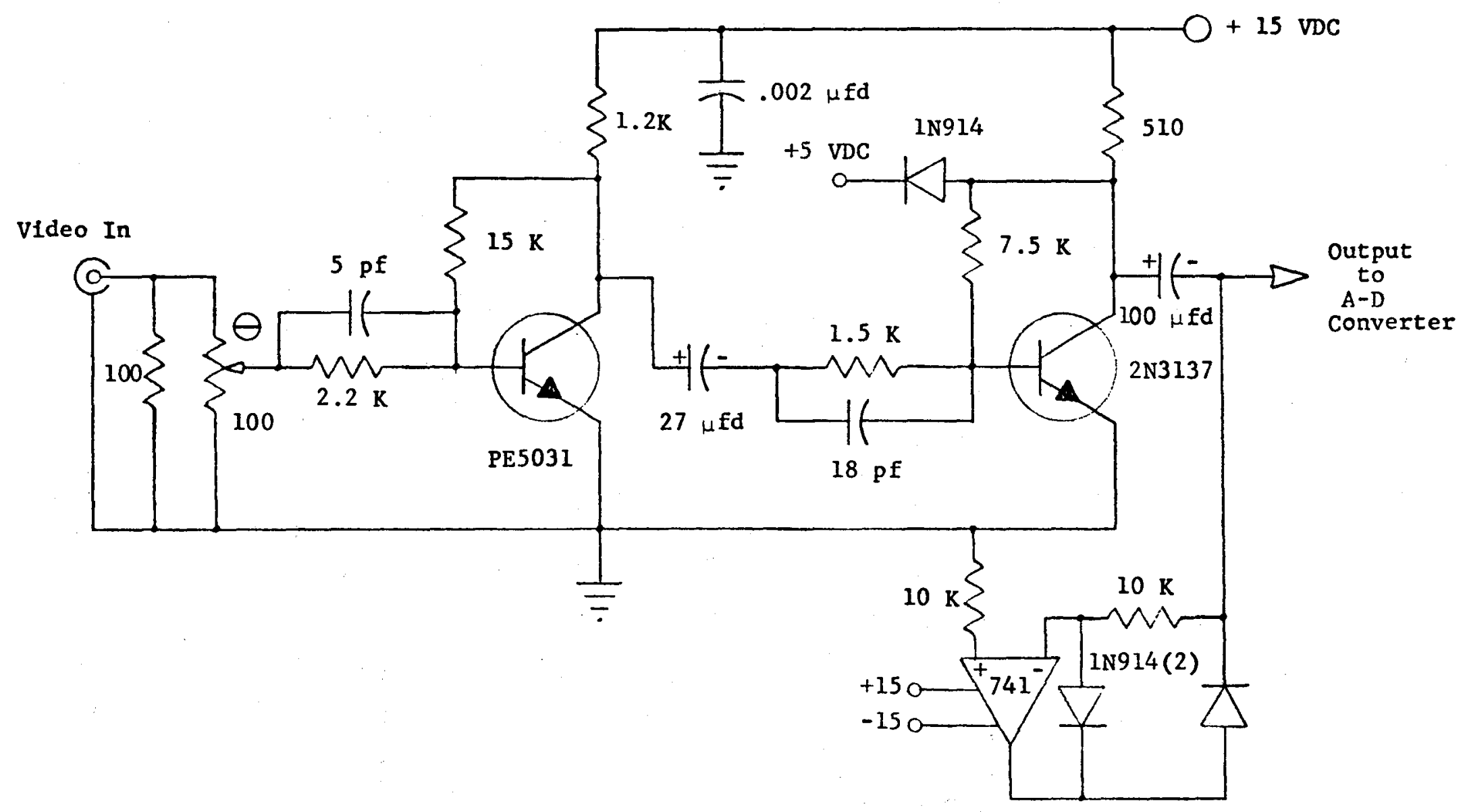

Figure 4. Echo Trap III Video Amplifier 


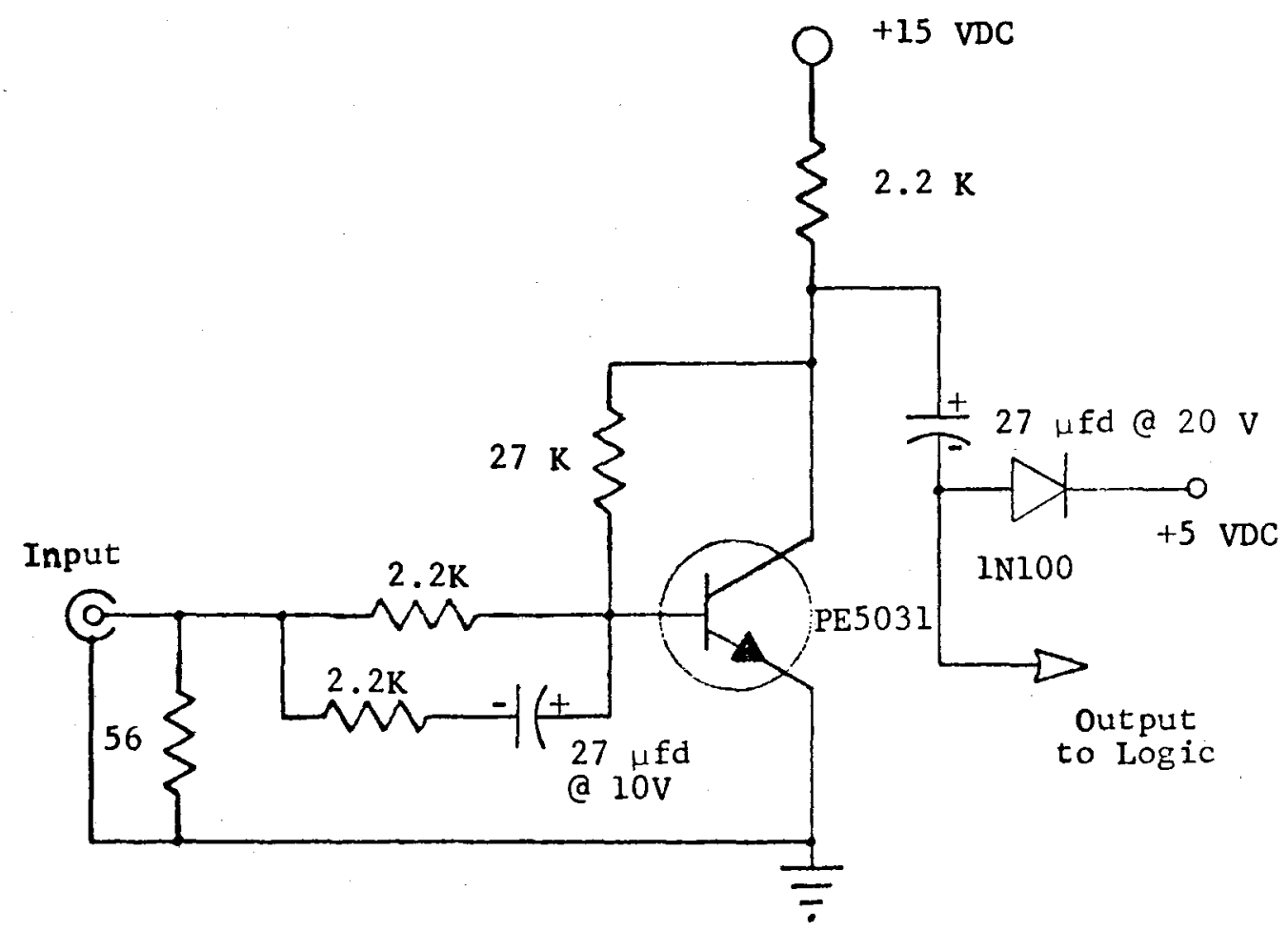

Figure 5. Echo Trap III IOI Amplifie:

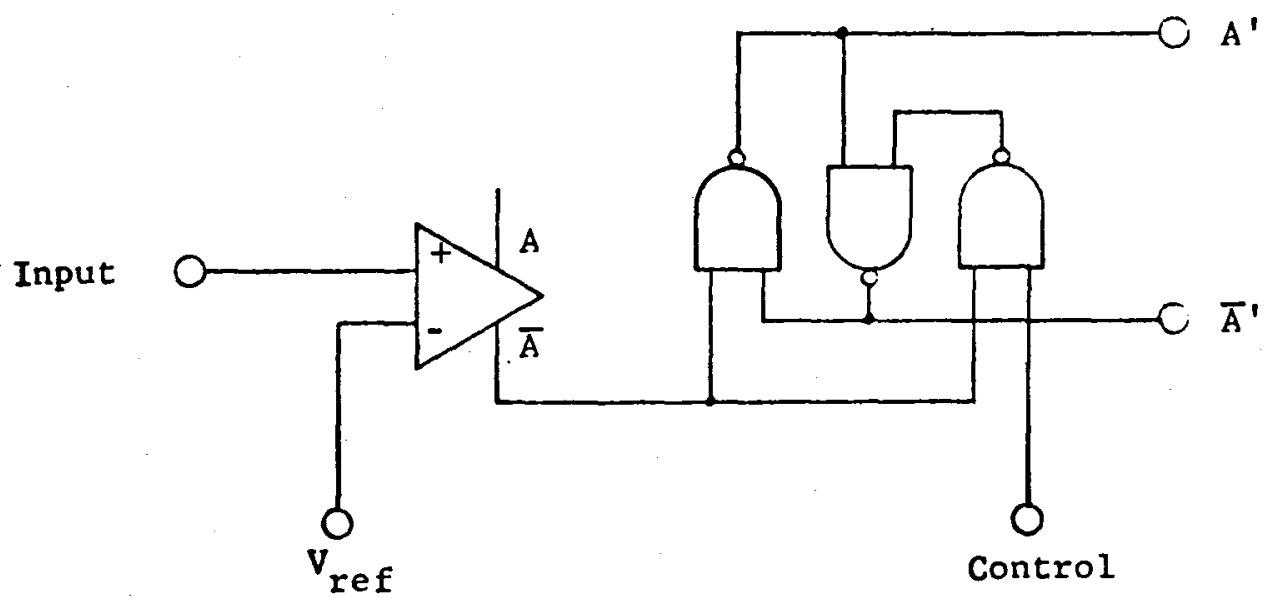

Flgure 6. Latching Comparator 
From the amplifier, the video goes to a special 4-bit A-D converter which is the heart of the system. This converter can either track the input signal or latch to its most positive value by means of a control line. Besides providing a 4-bit binary output, it also provides flaw level discrimination outputs for use by the control logic. To understand how this converter works, consider Fig. 6. Behind the comparator in Fig. 6 is a series of three 2-input NAND gates which comprise a controlled R-S flip-flop. The $\bar{A}$ output of the comparator is being used and will remain high until the input to the comparator exceeds the reference level. When this happens, the $\bar{A}$ output falls, and the $A^{\prime}$ and $\bar{A}^{\prime}$ logic outputs go from low to high and high to low respectively. If the control line is at $-5 \mathrm{~V}$, the outputs ( $A^{\prime}$ and $\bar{A}^{\prime}$ ) will toggle between states as the input goes above and below the reference level. However, if the control line is at $0 \mathrm{~V}$ when the input exceeds the reference, the outputs will latch with $A^{\prime}$ being high and $\bar{A}^{\prime}$ being low. The actual comparator output, $\bar{A}$, can still toggle between states but the logic outputs $A^{\prime}$ and $\bar{A}^{\prime}$ cannot.

If many of these comparators are used, each with the same input, and each one referenced to progressively higher voltage, a high speed parallel type A-D converter has been built that will retain peaks. Figure 7 shows the complete converter. Each comparator is referenced $100 \mathrm{mV}$ more positive than the one below it, with the bottom comparator being referenced to $100 \mathrm{mV}$. The input signal is applied to all of the positive comparator inputs in parallel. With this configuration, the circuit will hold the 
positive signal peak when in the latching mode. The circuit has 15 comparators yeilding 16 possible states including zero. These outputs are decoded, using Schottky-clamped TTL logic, to 4 binary bits. Some of the logic gates serve no purpose in the decoding, but are included to help equalize the propagation delay to the decoded output.

Consider the converter in the latching mode. If the input were to change abruptly from zero volts to $+620 \mathrm{mV}$ and back to zero, the bottom 6 comparators all toggled because their references were exceeded. Because of the latches however, the output will be frozen at 0110 , or binary 6 . This is exactly how the circuit operates on echoes.

Outputs are also provided from the bottom 5 comparators ahead of their latches. These go to selector switch SI to permit selection of the desired threshold. It is important to note that the resistor string that biases the comparators does not have to be linear (all equal resistors). These resistors may be weighted to produce a logarithmic output. Both techniques have been used, with a general preference for the log mode.

During the following descriptions it may be helpful to refer to the master logic schematic at the end of the report (Fig. 11). The alphanumeric designations refer to logic chips on that schematic. Also shown are the locations of the lettered waveforms used in the descriptions.

\section{Amplitude Determination}

Figure 8 shows a typical video input at $\mathrm{F}$. If the threshold selector is set on level 2, the output shown at $B$ is available to the control logic. The output from the IOI amplifier passes through a Schmitt NAND (J1) and results in signal A. A and $\mathrm{D}$ are combined in a NAND gate (RI) to yield signal C. Signal $C$ is the input to an 8-bit shift register (B2) whose outputs are 


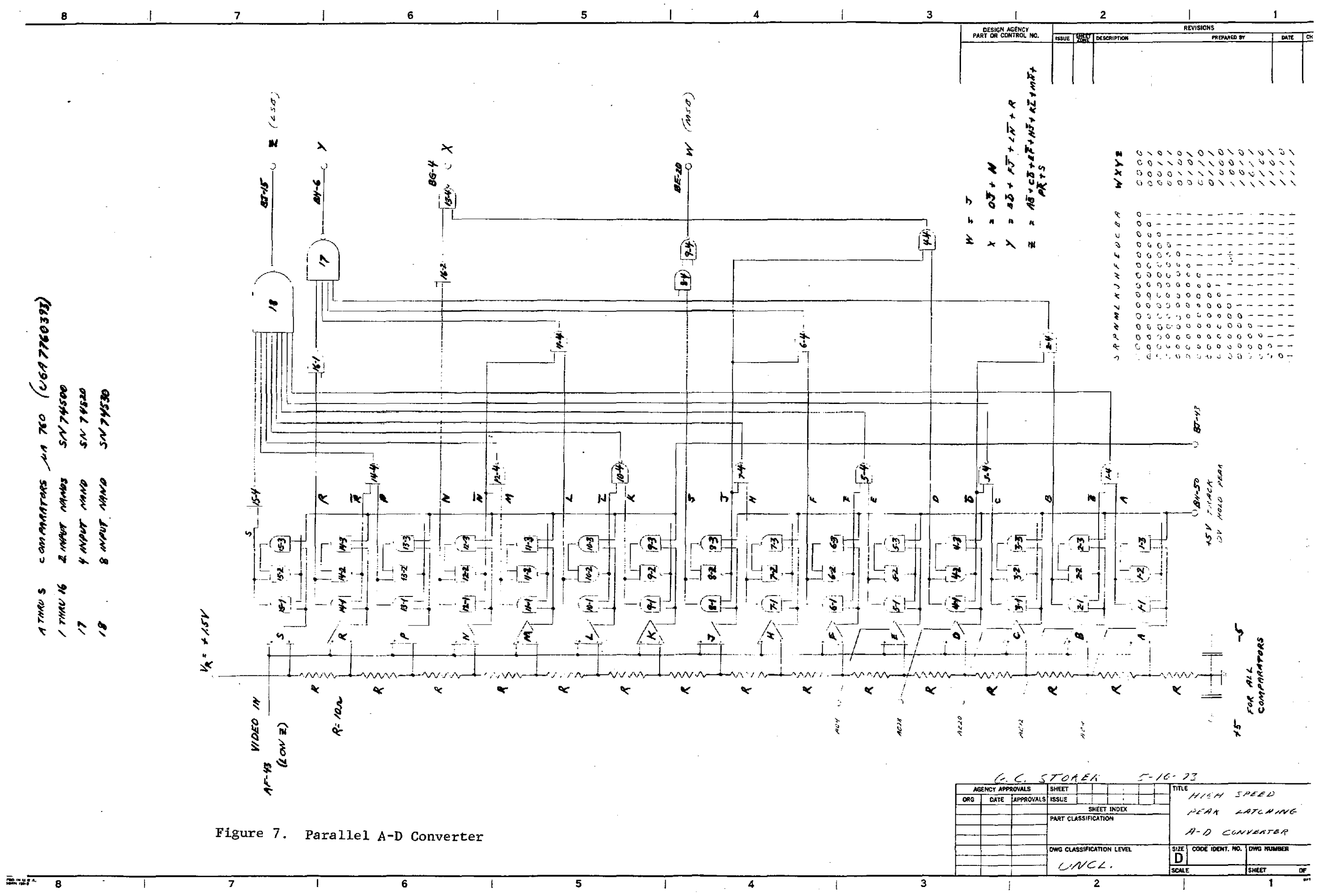




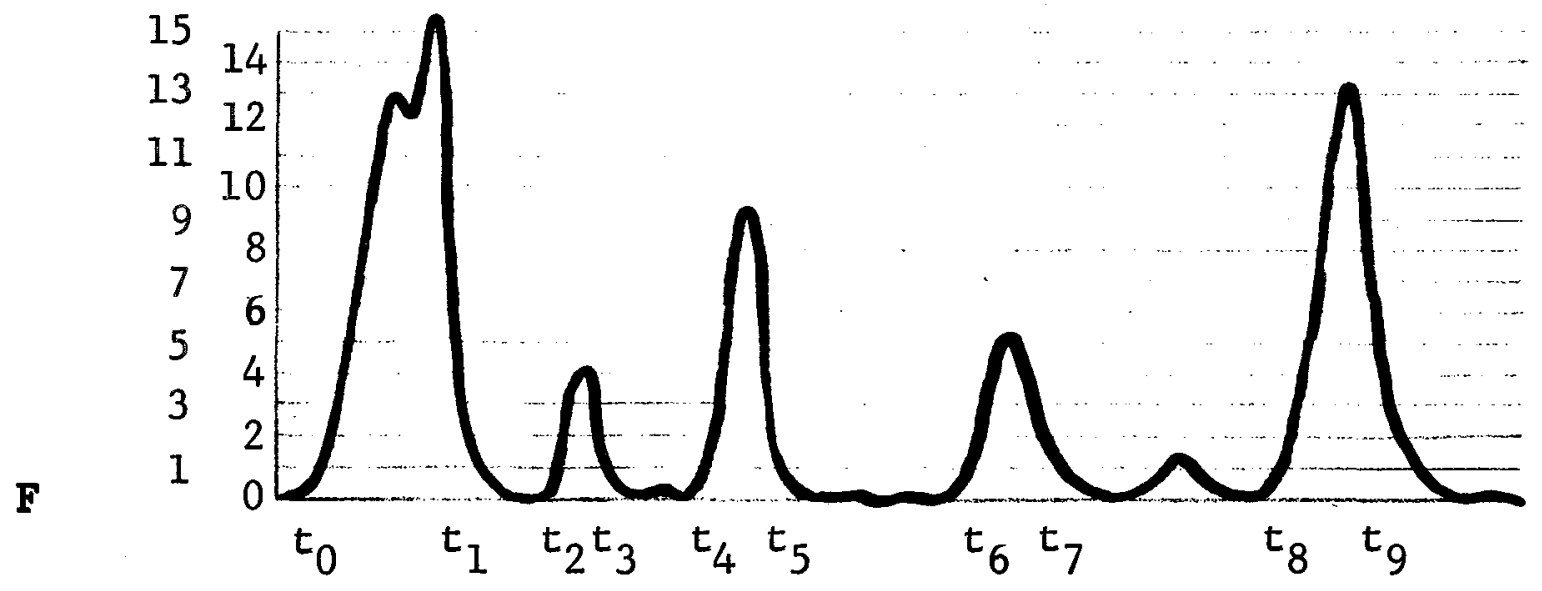

B
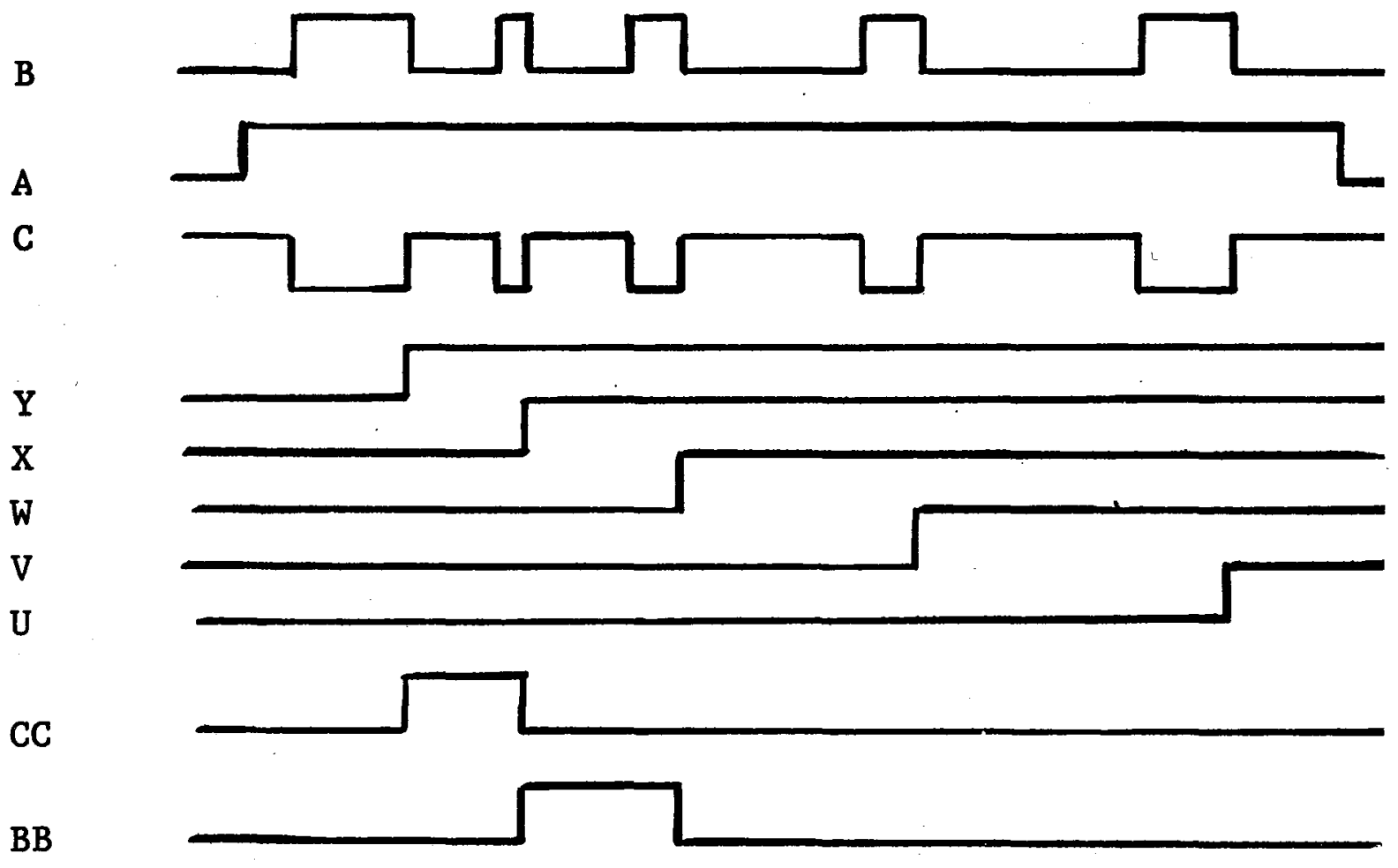

$A A$

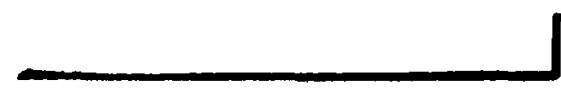

Z

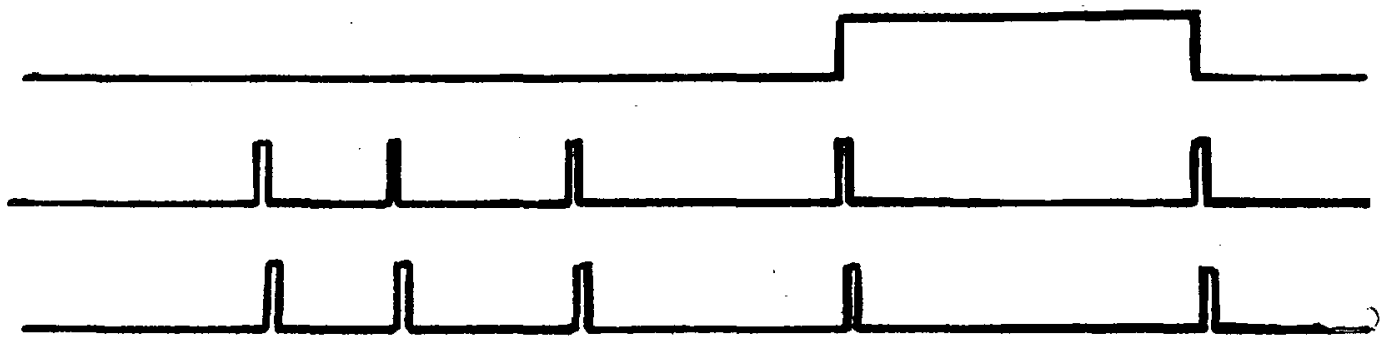

SS

Figure 8. Video Input and Digital Results 
shown as $\mathrm{Y}, \mathrm{X}, \mathrm{W}, \mathrm{V}$, and $\mathrm{U}, \mathrm{X}$ is inverted and combined with $\mathrm{Y}$ in an AND gate (KI) to yield output CC. In a similar manner, $X$, $W, V, U$ are combined to form outputs $B B, A A$, and $Z$. These outputs control four quad bi-stable latch circuits (E1 through E4). These latch circuits will store at their outputs whatever value the A-D converter holds at the time their inputs fall. Signal B is also fed to a one-shot (HI) yielding signal $\mathrm{T}$. Signal $\mathrm{T}$ also goes to a one-shot (HI), then through two NAND gates (R1) to yield signal SS. Signal SS is the latching control for the A-D converter. When SS is low, the A-D is in a peak latching mode. When SS is high, the $A-D$ is in a tracking or reset mode. Operation proceeds as follows: At $t_{0}$ the front surface echo crosses the threshold and since SS is low, the A-D converter is in the latching mode. The echo proceeds through its peak value then crosses the threshold again in the other direction at time $t_{1}$. At this point in time the $A-D$ holds the peak value of the front surface echo. Since this is not of interest, nothing is done with this value and the $A-D$ is reset approximately 40 nsec later by the first pulse seen on signal SS. At time $t_{2}$ the first flaw echo crosses the threshold, proceeds through its peak, then decreases below the threshold at time $t_{3}$. Now the $A-D$ contains the peak value of the first flaw signal. Since this is wanted information, this value is latched by the channel one bi-stable latch (E1). This is accomplished by signal CC falling at time $t_{3}$. Signal SS again resets the A-D and this operation repeats until the peak amplitudes of all four echoes shown are safety stored in the latches for future use. 
The signal SS is delayed from $t_{1}, t_{3}, t_{5}$, etc., approximately 40 nsec to allow time for the amplitudes to be stored in the latches. If more than 4 flaws had been received, only the first 4 would have been saved. If no flaws are received, no information would be stored. The amplitude information stored in the latches will be sent to the computer in a multiplexing cycle which will be described later.

\section{Depth Determination}

In order to measure the flaw delay time digitally, an accurate clock is necessary. This is provided by a $40 \mathrm{MHz}$ crystal controlled oscillator which passes through a Schmitt NAND (J1) and drives a 4-bit binary counter (A13). This provides time bases of $25,50,100,200$, and $400 \mathrm{nsec}$. The various time bases are front panel selected by means of switch $\mathrm{S} 2$.

All of the binary counters used are $7493 \mathrm{~A}$ chips. Since this counter is not specified to $40 \mathrm{MHz}$, some selection of chips may be required. It was found that the majority of the $7493 \mathrm{~A}$ chips tested would count $40 \mathrm{MHz}$ reliably. If selection is necessary, only chips $A 1, A 4, A 7, A 10$, and $A 13$ need be considered.

The first four signal waveforms in Fig. 9 are the same ones described in the section on echo amplitudes. Signal $\mathrm{C}$ is inverted (NI) to yield signal D. Signal D is the input to an 8-bit shift register ( $B I$ ) whose outputs are shown at $G, H, J, K$, and $L$. Output $\mathrm{H}$ is inverted (N1) and combined with output $\mathrm{G}$ (and clock pulses) in a NAND gate (Sl) to give output $Q$ in a similar manner, $\mathrm{J}, \mathrm{K}$, and $\mathrm{L}$ are combined with output $\mathrm{G}$ (and clock pulses) to 


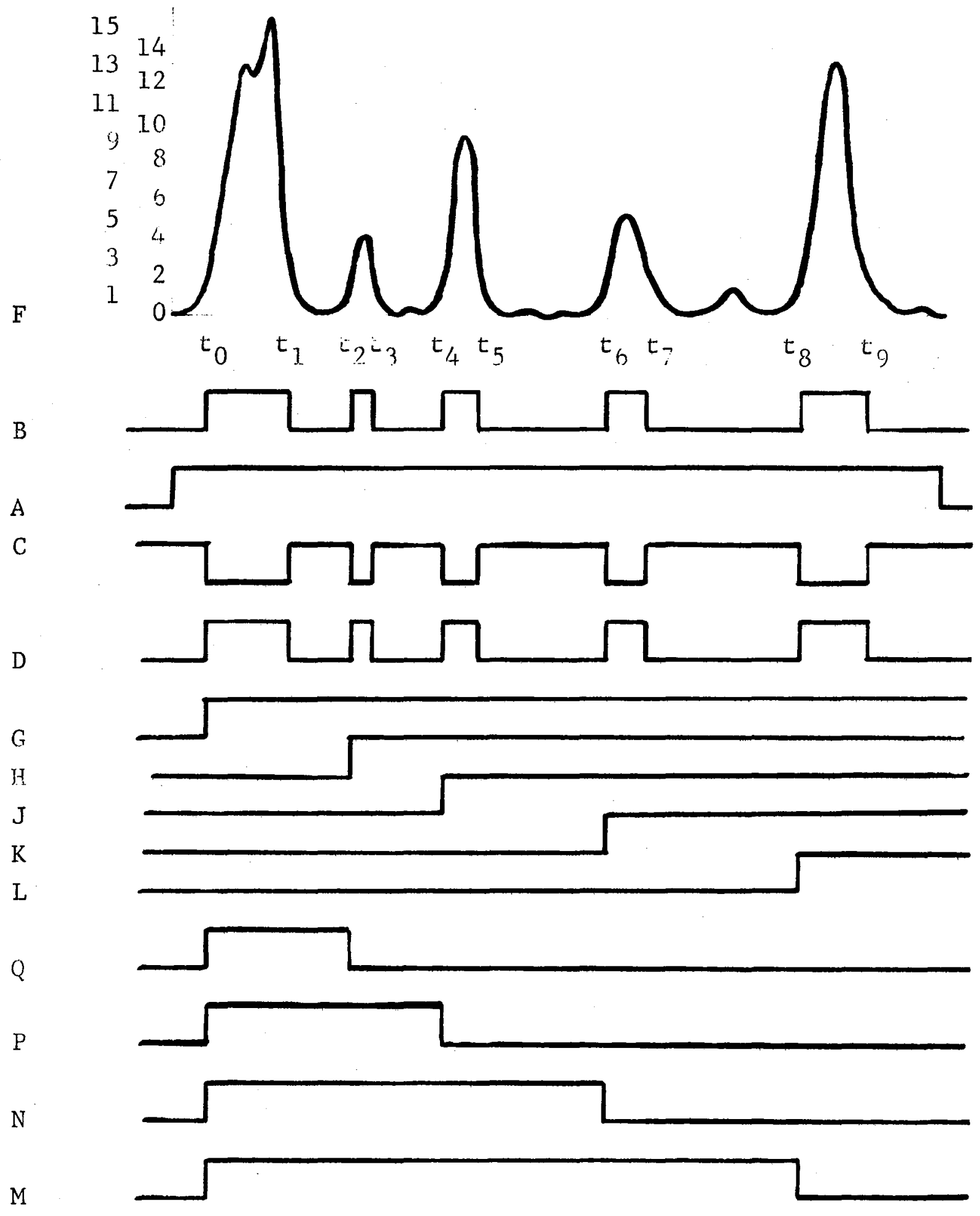

Figure 9. Depth Determinarion Wavt turms 
give outputs $P, N$, and $M$. The wave forms shown as $Q, P, N$, and $M$ are not correct as clock pulses have been deleted so that the timing interval is more clearly seen.

Signal $Q$ is the input to a 10 -bit binary counter comprised of three cascaded 4-bit binary counters (Al, A2, A3). Although this combination will count to 12 bits, only 10 bits are used. Each of the other outputs ( $P, N, M)$ drives a similar 10-bit counter.

In operation $t_{0}$ (the rise of the front surface) is used as a timing reference for all flaw channels. This can be seen as the simultaneous rise in signals $Q, P, N$, and $M$. All four binary counters are now counting clock pulses. At $t_{2}$ the first flaw arrives. As can be seen at signal $Q$ the gate is closed to channel one binary counter. This counter now holds the time to flaw \#1. If the velocity of propagation in the material being tested is known, this time is directly proportional to the depth of the flaw in the material. This operation continues with $P, N$, and $M$ closing at times $t_{4}, t_{6}$, and $t_{8}$, respectively. The four delay times stored in these counters will be sent to the computer during the multiplexing cycle.

\section{Multiplexing Cycle}

All multiplexing is done under interrupt control in the interface. One 16-bit data bus is used and the data from all four flaw channels is sequentially placed on this bus. An asynchronous approach is used so that critical timing is unnecessary. The output format for this 16-bit data word is shown below. 


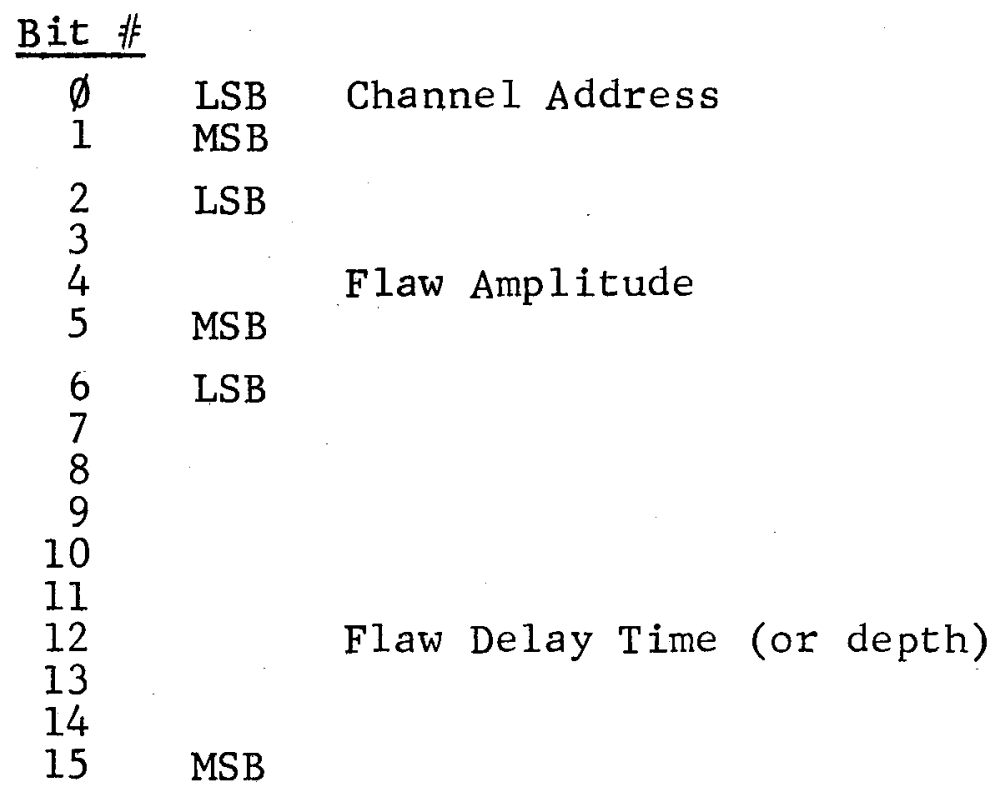

Figure 10 shows the multiplexing timing. Using a logic output or solid state switch in the computer, line SSS has been held low since the previous multiplexing cycle. Through a Schmitt NAND (J2) this holds signal DD high. This line goes into NAND gate T1. From a previous reset pulse (described later), binary counter Al4 is reset to all zeroes. The lowest 2 bits of this counter form the address for a 4-to-1 data selector (C8). Address 00 (channel 1 ) causes the signal level at $X$ to be applied to line $\mathrm{KK}$. $\mathrm{KK}$ also goes into NAND gate $\mathrm{Tl}$. If at least one flaw echo has been received, lines $X$ and $\mathrm{KK}$ will be high. The lowest 2 bits on Al4 also serve as the address fed to the computer (as $B \emptyset$ and $B l$ on the data bus) and to drive the other data selectors ( $\mathrm{Cl}$ through $\mathrm{C} 7$ ) which determine what channel is connected to the bus. Bit 3 of Al4 (at zero) is fed to an inverter (N2) and yields signal LL which enters gate Tl. IOI signal $A$ is inverted (NI) to become signal E, which also enters NAND gate T1. Finally, the IOI signal is buffered by two AND gates 


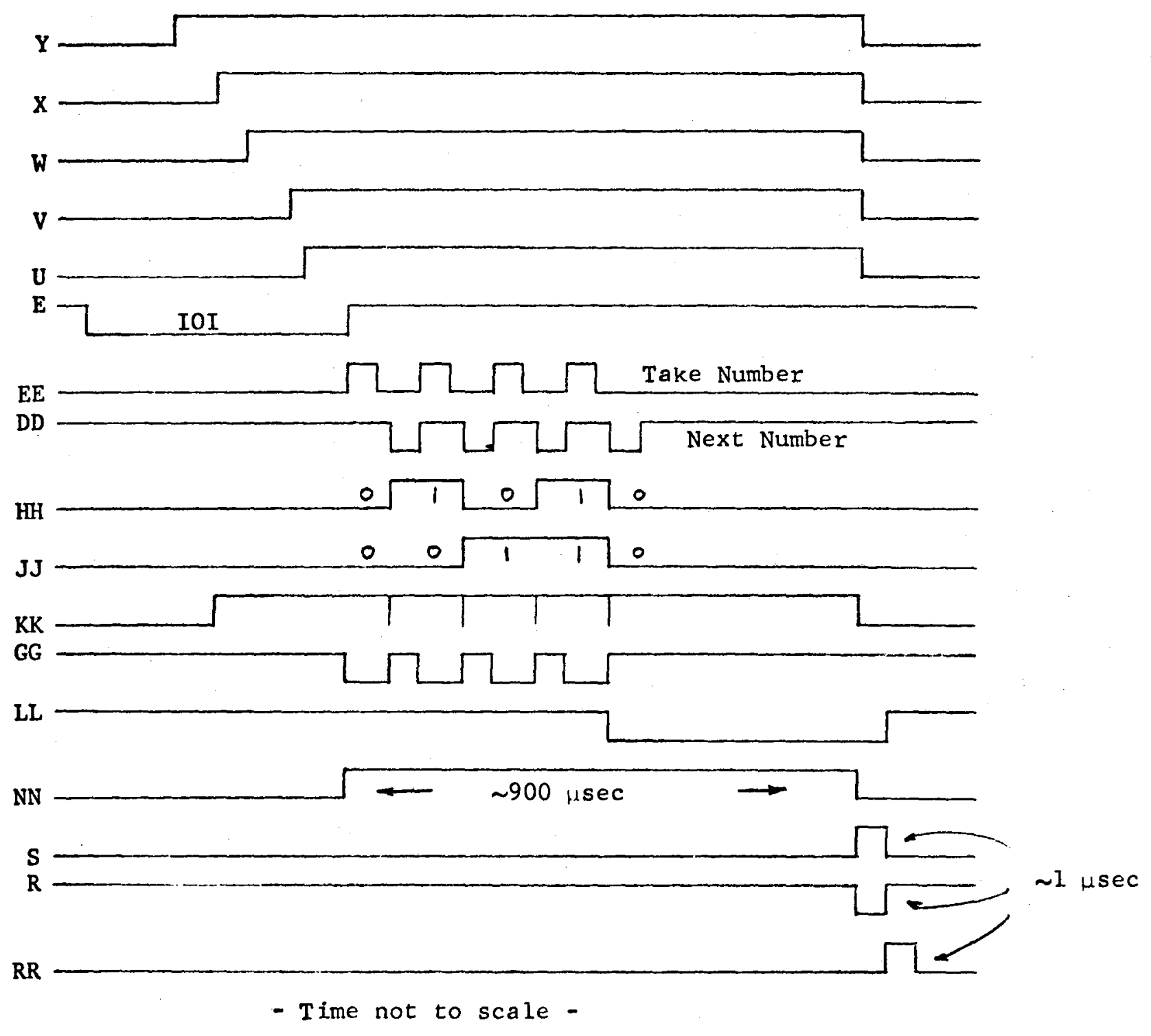

Figure 10. Multiplex Tuning

(K2) and connected to the data selectors (CI through C7). This will inhibit the selectors during the IOI.

When signal $E$ rises (end of IOI) the AND gates (K2) will enable the data selectors and place channel 1 amplitude and time delay information on the output bus. Also, Tl's output 
will fall (signal GG) and trigger a one-shot (H3) which generates the first interrupt (signal $\mathrm{EE}$ ). This interrupt goes to the computer signifying that data is on the bus and ready to be read. Had there been no flaw echoes, lines $\mathrm{X}$ and $\mathrm{KK}$ would be low and no interrupt would have been generated. The interface is now waiting for the computer.

After the data has been read and stored in memory, the computer first drives line sSs high, and then low. This, in effect, asks the interface for the next data word. SSS going high causes DD to fall. When DD falls two things happen. First, counter A14 is incremented to output address 01 . Through C8, this causes signal $W$ to be transferred to line $\mathrm{KK}$. At the same time all channel 2 information is put on the bus. Secondly, DD falling inhibits gate $\mathrm{Tl}$ causing its output (GG) to rise.

When DD rises again (about 1 usec later) output GG will fall if there was a second data word to output (signal $\mathrm{W}$ being high). If it does fall, the second interrupt will be generated by $\mathrm{H} 3$. This process continues until there are no more flaws or until all four channels have been read. If all four are read, the computer will ask for a fifth channel. When it does, counter Al4 goes to address 100. That third bit being a one causes line LL to go low, which inhibits any further interrupts from being generated. Essentially, the interface denies any more data. The computer software is written such that if no interrupt is received within one machine cycle (approximately $I \mu s e c$ ) after line SSS is pulsed, it knows no more data is available until the next excitation pulse. 


\section{Reset Cycle}

Figure 10 also shows the reset timing. The IOI signal drives one-shot $\mathrm{H} 2$ such that line NN will be high for approximately $900 \mu \mathrm{sec}$. When NN falls, one-shot H2 will generate a 1 usec pulse (lines $\mathrm{S}$ and $\mathrm{R}$ ). Line $\mathrm{R}$ will then clear the twoshift registers $\mathrm{B} 1$ and $\mathrm{B} 2$. Line $\mathrm{S}$ drives another one-shot (He) which generates signal RR and this signal resets counter Al4 and all four binary time counters (AI through A12). Resetting A14 after the shift registers have been cleared guarantees that no spurious interrupt will be generated as a result of a time race problem with $\mathrm{A} 14, \mathrm{C} 8$, and $\mathrm{B} 2$.

After line $\mathrm{RR}$ falls the interface is ready for the next IOI pulse. The $900 \mathrm{usec}$ delay is the maximum time allowed for the multiplexing cycle. The actual time required is almost solely determined by the computer system and its software. The interface alone is capable of cycling all four channels in less than $10 \mathrm{usec}$. The actual maximum repetition rate for the ultrasonic system is limited by the $900 \mathrm{usec}$ delay plus the total IOI time. If the IOI is less than $100 \mathrm{usec}$, the system may be pulsed approximately 1000 times a second.

This concludes the operational description. The master logic schematic (Fig. 11), power supply schematics (Fig. 12), and photographs of the interface (Figs. 13 and 14) follow. 


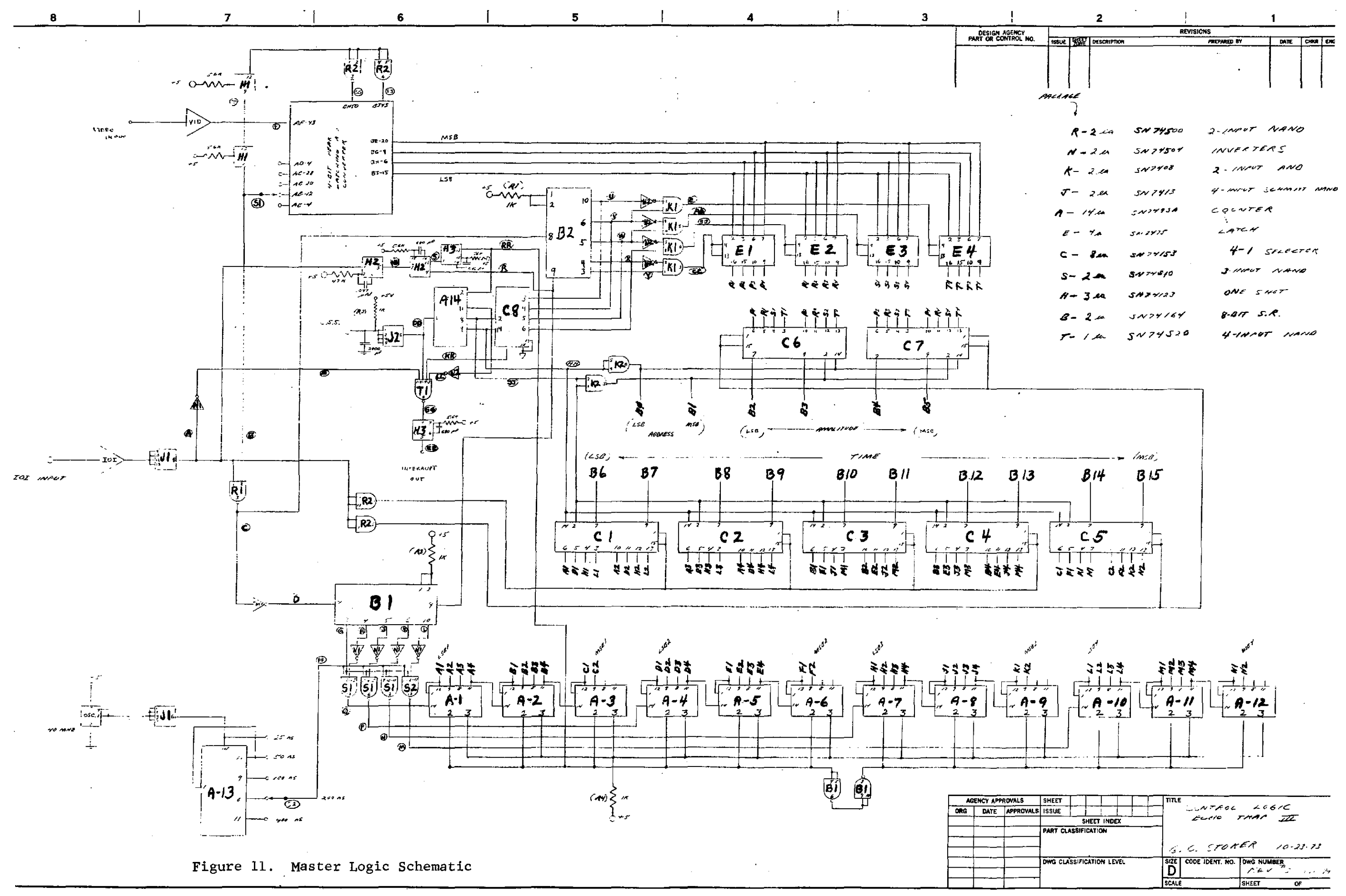




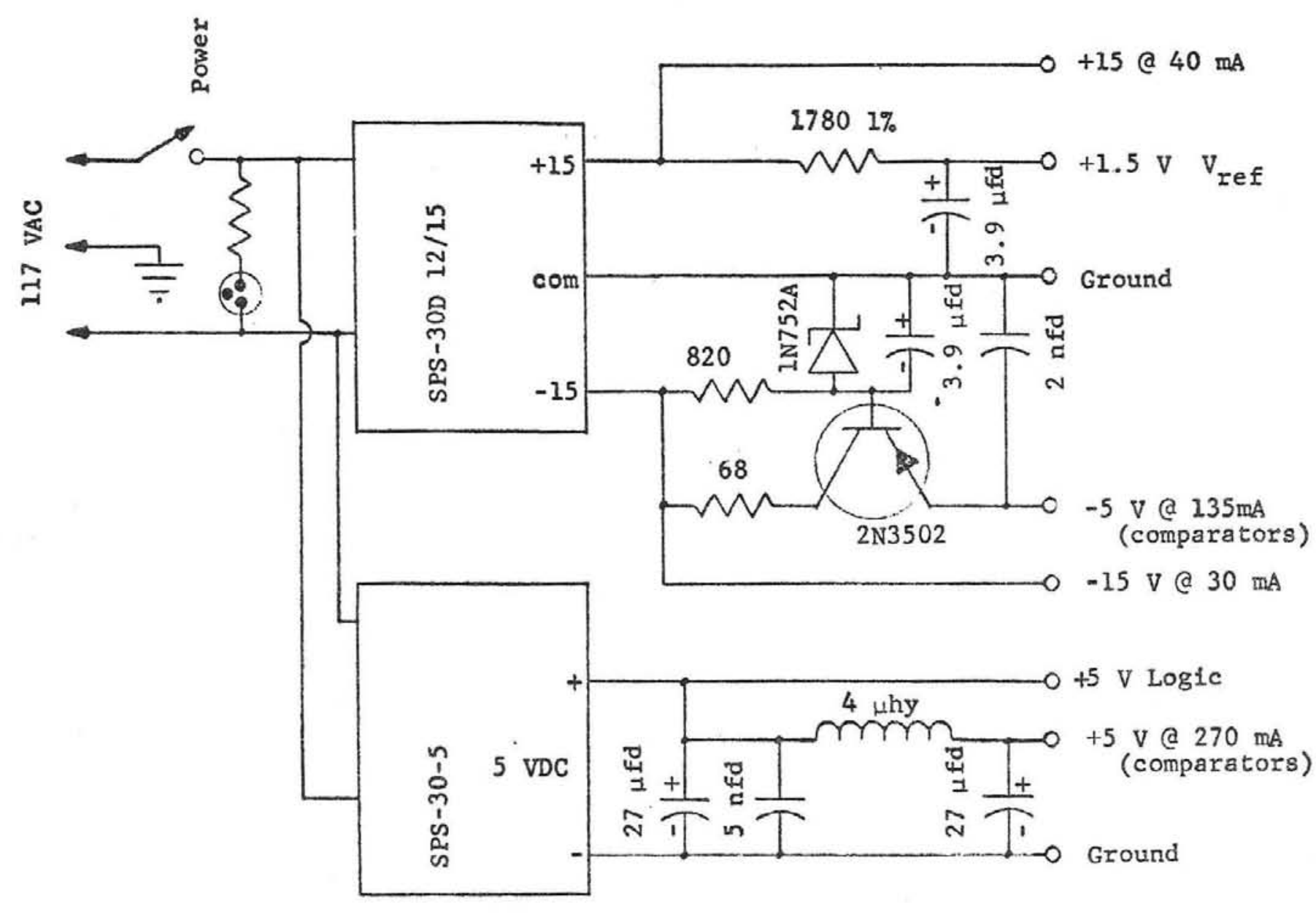

Figure 12. Power Supplies 


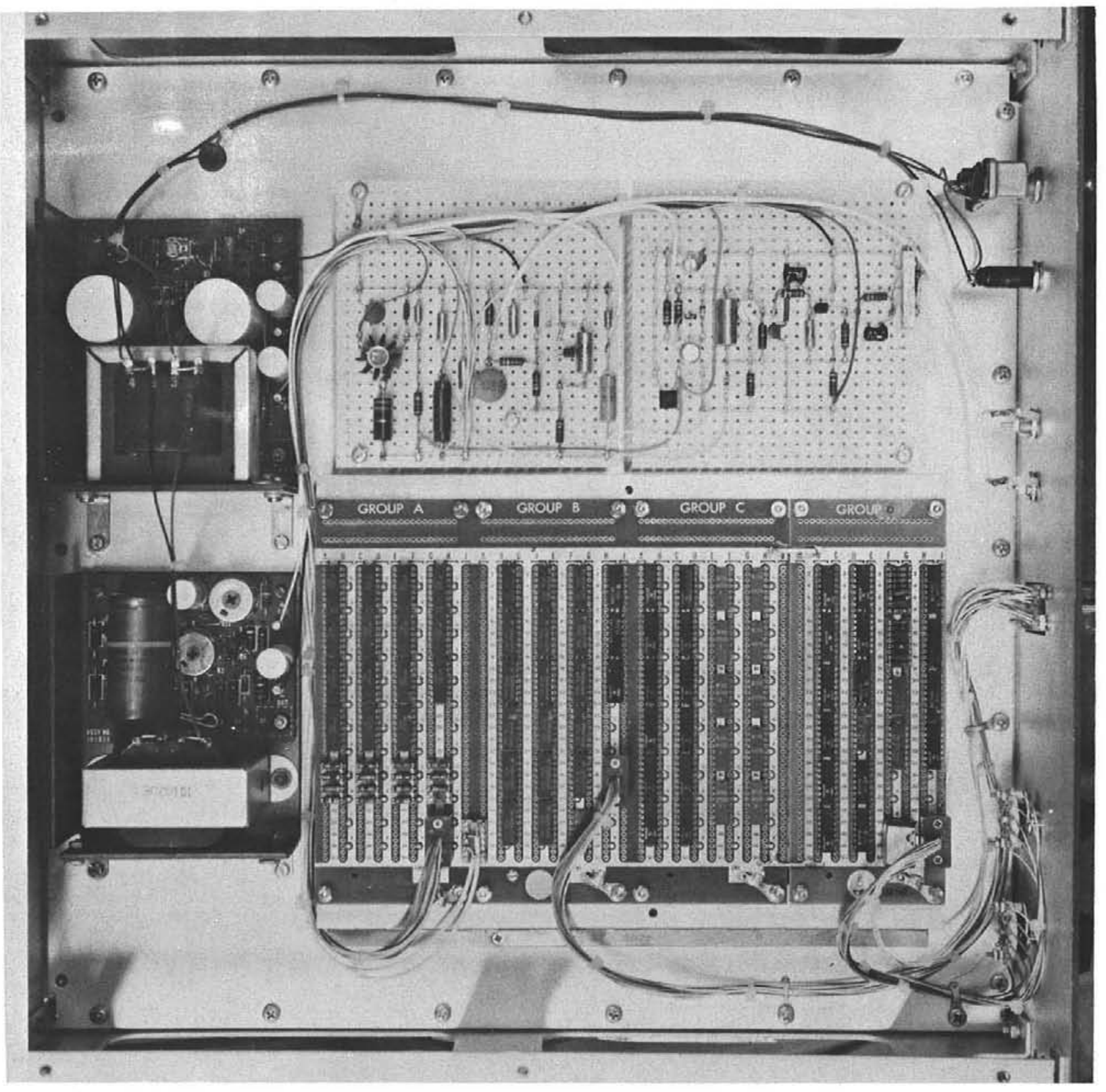

Figure 13. Top View Echo Trap III 


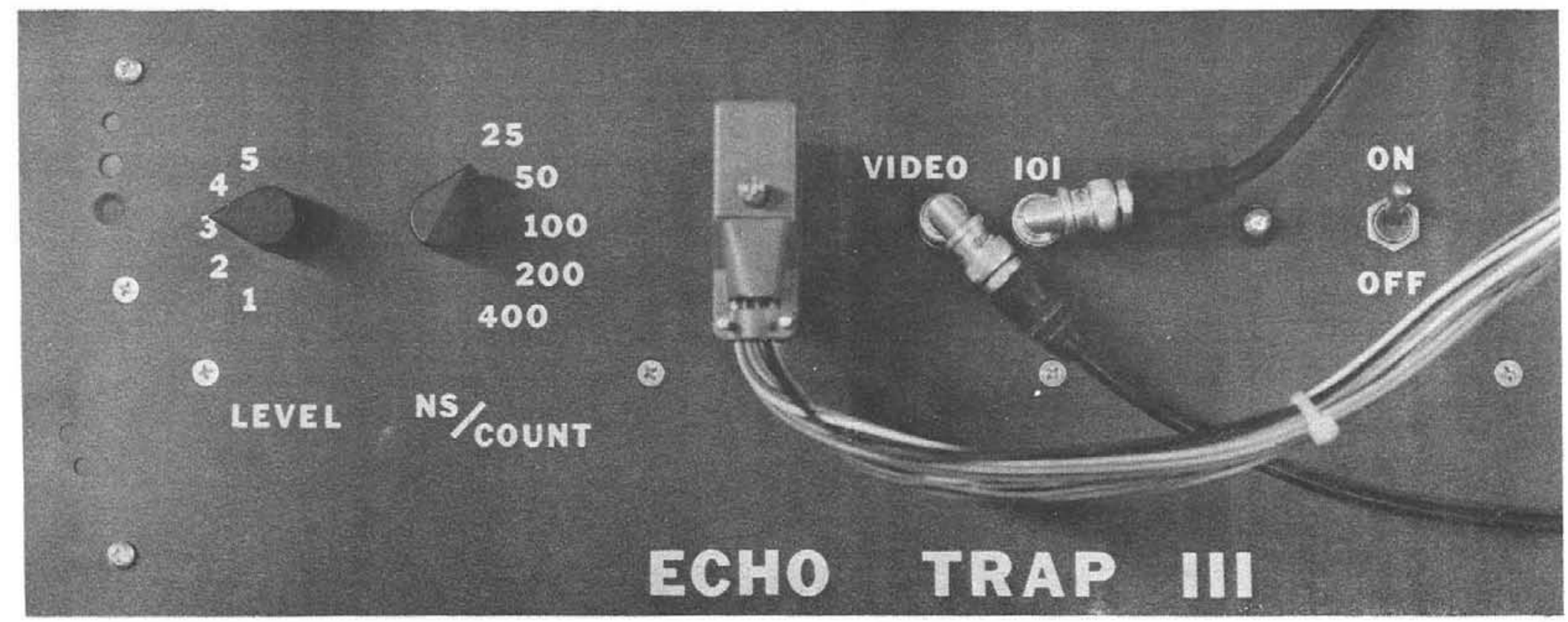

Figure 14. Front View Echo Trap III 
Distribution:

Lockheed Space and

(3) Missile Center

Sunnyvale, CA 94087

For: C. Hutchison, SPL-32

R. Foster, LMSC 81-20

R. Crist, LMSC 84-35

Lawrence Livermore Laboratory

PO Box 808

Livermore, CA 94551

For: Keith McMillan, L-415

B. 0. Bolden, L-349

Dana Elliott, M-I

Los Alamos Scientific Laboratory

PO Box 1663

Los Alamos, NM 87544

R. W. McClung

Oak Ridge National Laboratory

PO Box X

Oak Ridge, TN 37830

L. E. Burkhart

Union Carbide Corporation

Y-12 Plant

PO Box Y

Oak Ridge, TN 37830

A. H. Gardner

General Dynamics

PO Box 748

Ft. Worth, TX 76101

Commanding officer

Naval Weapons Station

Attn: Harvey LeGrone

Seal Beach, CA 90740

1540 T. B. Lane Attn: J. Freedman, 1542

1535 R. D. Christopher

1537 B. E. Bader

2400 R. S. Claassen

5130 G. A. Samara

5160 W. Herrmann Attn: B. M. Butcher, 5167

5600 A. Y. Pope

5628 S. McAlees

5720 M. L. Kramm

5725 D. J. Rigali

5725 J. L. Irwin

5800 L. M. Berry

5840 D. M. Schuster

8110 A. N. Blackwell

8266 E. A. Aas (2)

8300 B. F. Murphy

8344 A. R. Willis

9300 L. A. Hopkins, Jr.

9320 M. Cowan

9350 F. W. Neilson

9351 D. W. Ballard

9352 0. J. Burchett

9352 J. H. Gieske

9352 G. C. Stoker (15)

9500 L. M. Heilman

3141 L. S. Ostrander (5)

3151 W. F. Carstens (3)

For: ERDA/TIC

(Unlimited

Release) 